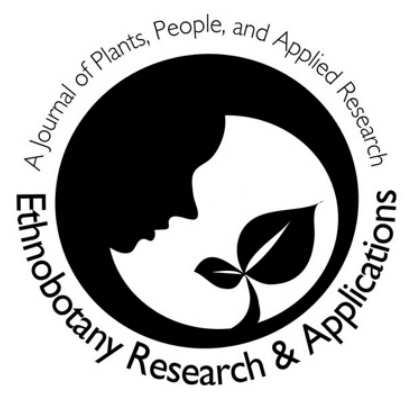

\title{
Folk medicinal plants used for the treatment of gynecological disorders by the rural population of Zorlu village (Turkey)
}

Arzu Ergül Bozkurt

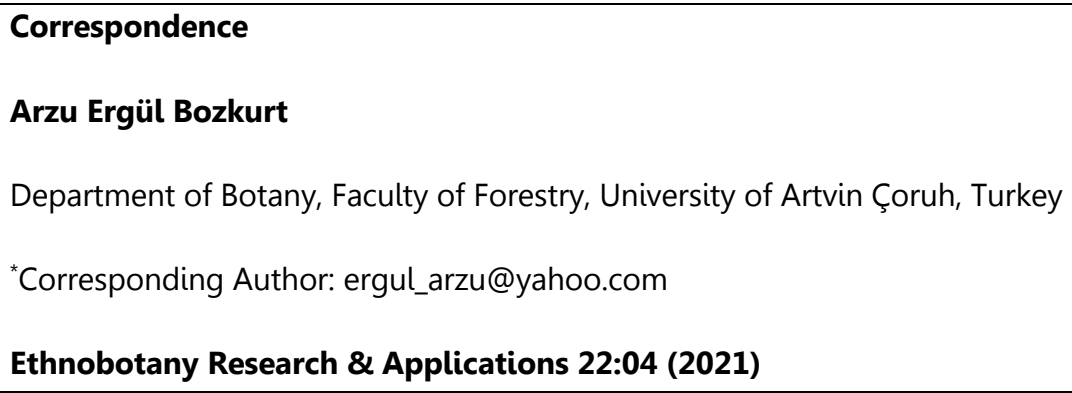

\section{Databases and Inventories}

\begin{abstract}
Background: This research deals with the indigenous medicinal plants used by rural population of Artvin-Borçka (Zorlu village), for the treatment of gynecological disorders. For this purpose, ethnomedicinal survey of Zorlu village in Turkey was carried out during 2008-2009.
\end{abstract}

Methods: In the present study, a two-part survey was made. The first part of the survey was determined the folk medicine used for treatment of gynecological disorders. Second part of the survey were determined the ethnomedicinal uses of the plants.

Results: Local inhabitants are extremely knowledgeable about the utilization of indigenous flora of the study area and knowledge about the treatment of gynecological disorders. As a result of the study 74 plant taxa belonging to 59 genera and 30 families were determined. These 74 plant taxa used the treatment of gynecological disorders. Information on 74 plant taxa with their botanical name, pharmacological activity, ethnomedicinal usage knowledges were provided. In addition, the pharmacological properties of these plant taxa such as antioxidant, antimicrobial, antibacterial, antitumor, antiviral, anxiolytic, antidepressant, anthelmintic, antimalarial, antidiabetic, anti-inflammatory, analgesic, tumor-inhibitory, antiallergic, antihepatotoxic, antiulcer, anticandidal, antifungal, anticancer, antibiotic, anticariogenic, antigenotoxic, antirheumatic, antipruritic and antihypertensive activities are presented in this study.

Conclusions: Present study is about the utilization of medicinal plants in Zorlu Village, they were used the traditional knowledge for the treatment of gynecological disorders. Documentation of such ethnomedicinal data on biological resources can be used for medical and pharmaceutical science.

Keywords: Turkey, medicinal plants, ethnomedicine; gynecological disorders.

\section{Background}

Plants are used for the treatment of many illnesses since a very long time. A wide range of herbal traditional medicines are used for the treatment of gynecological disorders. Traditional ethnomedicine has advantage in treating gynecological disorders due to lack of awareness and shyness. Medicinal plants are the basis of many of the modern pharmaceuticals we used today for the treatment of various ailments (Abraham 1981, Atal \& Kapur 
1982, Shukla et al. 2008). A large ratio of such medicinal compounds was discovered with the aid of ethnomedicinal knowledge of their traditional uses (Krishnaraju et al. 2005). And medicinal plants and plant-derived medicines are widely used in the world (Johns et al. 1990, Hamayun et al. 2003).

Ancient people mainly depend on medicinal plants for their health. Historically, all medicinal preparations such as extracts, mixtures, etc. were derived from plants. On the other hand, in the simple form of raw plant materials were also used for the treatment of some ailments. (Farnsworth \& Soejarto 1991). The local people acquired the knowledge of medicinal plants by methods of trial and error. After these determinations, they became the storehouse of knowledge of useful and harmful plant taxa. Due to erosion of traditional cultures, these rich unwritten local knowledge on uses of medicinal plants would be lost, for that reason, this ethnomedicinal usage of the plants must be properly documented and preserved. (Rama Rao \& Henry 1996, Qureshi et al. 2010).

In this study aim to transfer of knowledge about the traditional usage of medicinal plant taxa and their treatment of gynecological disorders in Zorlu village of Turkey. The extract, which was prepared with using medicinal plants for the treatment of gynecological disorders used by the local people of Zorlu village, was brought to light. Because the last representative (the author's close relative) is very old and with the concern about this invaluable information will be lost, Zorlu village has been determined as a field of study. In addition, the determination of the medicinal plants, which can be used ethnomedicinal purposes, is most important issue because this information will return to contribute to the economy of the local people. In this way, migration from village to city can be prevented and the use of medicinal plants can be expanded.

\section{Materials and Methods}

\section{Study area}

Zorlu village is located in the Artvin-Borçka region of Turkey. This village has most of local inhabitants from Georgian origin, the Georgian language is still widely spoken there. Therefore, the names of some plant taxa were named as Georgian, in this survey. Zorlu village belongs to the Euro-Siberian plant geography region and falls within the A8 grid square according to the grid classification system developed by Davis (1965-1985). The geological structure of the research area consists of rocky slopes. The soil groups of research area are brown forest soils, colluvial soils and high mountain meadow soils in general. The main vegetation types in the research area are Forest, alpine, subalpine, and rocky. Forest vegetation mainly includes the tree formation of plant taxa such as Abies nordmanniana (Stev.) Spach, Picea orientalis (L.) Link, Pinus sylvestris L., Fagus orientalis Lipsky, Populus tremula L., Carpinus betulus L. (Atalay 1983; TKH 1990; Anonymous 1994). The climate of the region is generally cool and rainy in summer, cold and snowy in winter (Anonymous 2002; Kolayli \& Şahin 2007). Climatic data were obtained from the Trabzon Agency of Meteorology. Average temperature in 2008-2009 years were $12.4^{\circ} \mathrm{C}$ and $12.8^{\circ} \mathrm{C}$, respectively. Total precipitation in 2008-2009 years are $600.4 \mathrm{~mm}$ and $888.2 \mathrm{~mm}$, respectively.

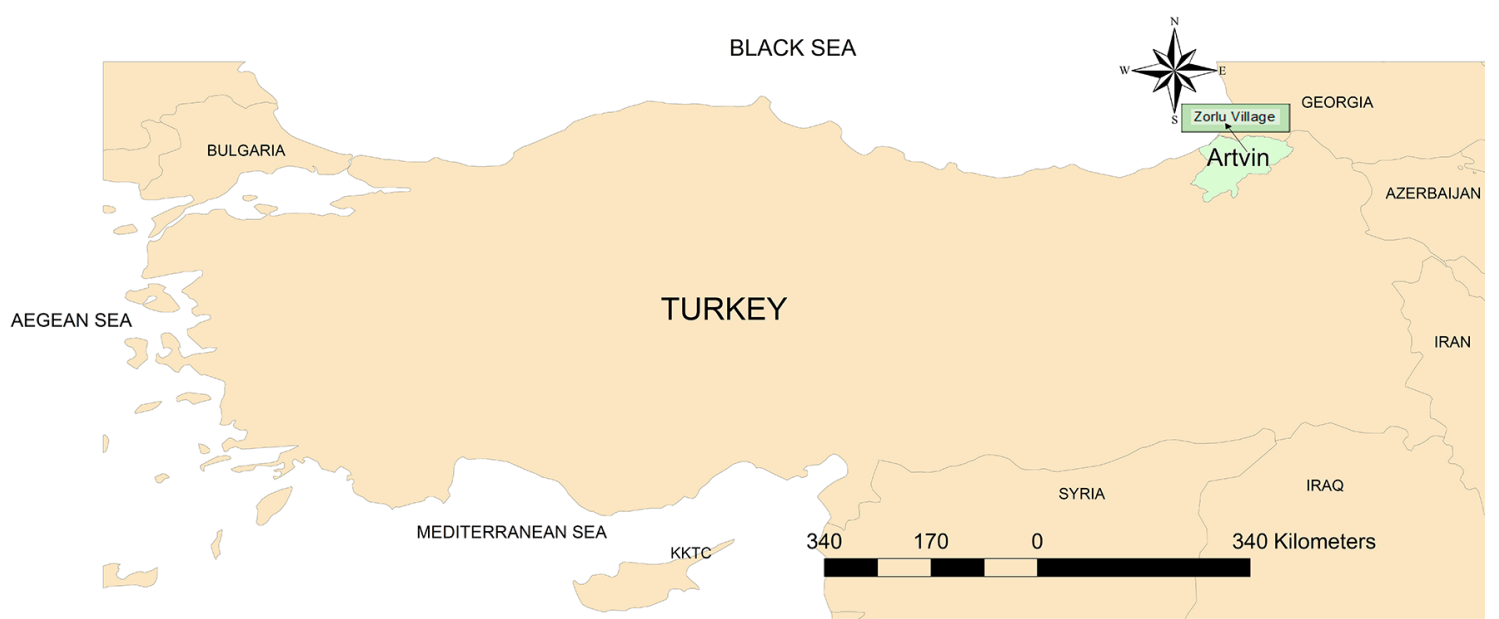

Figure 1. The illustration of Artvin-Borçka (Zorlu village) location in Turkey

\section{Material collection and statistical analysis}

This study was carried out between 07.04.2008-07.04.2009 and more than 200 plant specimens were collected. Plant samples were collected in accordance with herbarium standards. At least one plant sample was prepared and deposited at Herbarium of Karadeniz Technical University Faculty of Forestry (KATO). Plant specimens were 
identified using "Flora of Turkey and the East Aegean Islands" (Davis 1965-1985). The Turkish names of plant specimens were given according to Güner et al. (2012). Information on the uses of these plants was obtained by face-to-face interviews with local people and was recorded using a survey form. In the survey form was included some information such as age, gender, education level, and occupation of the persons. In addition, it was determined that which plants selected by local people and how they used these plants for the treatment of disorders. While providing information about plants by local people, it was provided that to show the plant samples in the nature by them. In the study, a two-part survey was done total of 58 informants. The first part of the survey was determined the folk medicine used for treatment of gynecological disorders. Second part of the survey were determined the ethnomedicinal uses of the plants. Table 1 is showed the information about the informants.

Table 1. Characteristics of informants

\begin{tabular}{llll} 
& \multicolumn{1}{c}{ Features } & $\begin{array}{l}\text { Number of } \\
\text { informants }\end{array}$ & $\begin{array}{l}\text { Percentage } \\
(\%)\end{array}$ \\
Gender & Male & 17 & 29.31 \\
Educational level & Female & 41 & 70.68 \\
& Illiterate & 7 & 12.06 \\
& Primary school & 28 & 48.47 \\
& Secondary school & 6 & 10.34 \\
Age groups & High school & 12 & 20.68 \\
& University & 5 & 8.62 \\
& $25-35$ & 4 & 6.89 \\
Occupation & $35-45$ & 10 & 17.24 \\
& $45-55$ & 31 & 53.44 \\
& $>55$ & 13 & 22.41 \\
& Worker & 7 & 12.06 \\
& Farmer & 9 & 15.51 \\
& Artisan & 4 & 6.89 \\
& Retired & 8 & 13.79 \\
& Housewife & 23 & 39.65 \\
& Student & 2 & 3.44 \\
& Self-employment & 5 & 8.65
\end{tabular}

The Factor of Informant Consensus (FIC) was used for the determining the homogeneity of information about the ethnomedicinal usage of the plants. And for determining the Use Value (UV), the number of plants usage and the number of informants was evaluated. FIC value ranges from 0 to 1 and 1 indicates the highest level (Trotter \& Logan 1986; Camejo-Rodrigues et al. 2003; Tardío \& Pardo-de-Santayana 2008; Giday et al. 2009:

$$
\mathrm{FIC}=\mathrm{Nur}-\mathrm{Nt} /(\mathrm{Nur}-1) \text { and } \mathrm{UV}=\mathrm{U} / \mathrm{N}
$$

Nur: the number of use reports of the plants by informants $\mathrm{Nt}$ : the number of taxa which were used any disease or disease group $U$ : the number of usage reports for any plant $\mathrm{V}$ : the number of informants

\section{Extract preparation}

Gynecological treatment method, which is used for pregnancy, was explained by Pakize Altuntaş (author's grandmother). Her mother (1940-1965 years) and her grandmother (1900-1930 years) were used this method for generations. But the last one to implement this method is her. She is explained that "Collected the 74 plant taxa, which is the scope of the study, are boiled in a barrel. The water must be lukewarm. The same water is used the next three days morning and the water is being used by heating. Patient should stay in the water for at least 1 hour". She was application this method between 1978-1992 years. As a result of this method, 10 patients had been cured and this method resulted pregnancy for them

A literature survey was carried out about the pharmacological properties of determined plant taxa, such as antioxidant, antimicrobial, antibacterial, antitumor, antiviral, anxiolytic, antidepressant, anthelmintic, antimalarial, antidiabetic, anti-inflammatory, analgesic, tumor-inhibitory, antiallergic, antihepatotoxic, antiulcer, anticandidal, antifungal, anticancer, antibiotic, anticariogenic, antigenotoxic, antirheumatic, antipruritic and antihypertensive activities. Because it is believed that the curative effect of these properties on extract. 


\section{Results and Discussion}

This study showed that the combination (extract) of 74 medicinal plant taxa used for the gynecological disorders were recorded and documented. These plant taxa are; Achillea biserrata, Acinos arvensis, Agrostemma githago, Ajuga reptans, Alyssum murale subsp. murale var. murale, Anagallis arvensis var. arvensis, Aristolochia pontica, Astragalus caucasicus, Bellis perennis, Buglossoides arvensis subsp. sibthorpiana, Cardamine hirsuta, Cichorium intybus, Cistus creticus, C. salvïfolius, Convolvulus cantabricus, Echium vulgare subsp. vulgare, Fragaria vesca, Genista tinctoria, Geranium columbinum, G. molle, G. purpureum, Hedera helix, Hypericum orientale, Juglans regia, Medicago lupulina, Onosma sericeum, Papaver lateritium subsp. lateritium, Plantago major subsp. major, Potentilla argentea, P. crantzii var. crantzii, P. recta, P. thuringiaca, Primula acaulis subsp. rubra, Ranunculus cappadocicus, Reseda luteavar. lutea, Rhododendron luteum, Rubus idaeus subsp. idaeus, Sambucus ebulus, Scabiosa columbaria subsp. columbaria var. columbaria, Sedum hispanicum, Senecio vernalis, Stachys annua subsp. annua var. annua, Stellaria media, Teucrium flavum subsp. hellenicum, T. polium subsp. polium, Thymus nummularius, T. praecox subsp. grossheimii, T. praecox subsp. skorpilii var. skorpilii, T. vulgaris, Trifolium dubium, Trifolium pratense var. pratense, Urtica dioica subsp. dioica and Vaccinium arctostaphylos. The usage plant parts are generally above ground, only the roots of Aristolochia pontica used in prepared extract for the treatment of gynecological disorders. The most applied sections of plants are above ground (73) and root (1) for the treatment of gynecological disorders.

In this study, 41 plant taxa (55.40\%) had have the ethnomedicinal importance. Many studies have been conducted to investigate the chemical composition of these plant taxa. Table 2 showed that the list of ethnomedicinal usage of these plant taxa.

As a result of the study, it was determined that the highest use value (UV) is found in Bellis perennis (0.68) followed by Cardamine hirsuta (0.43), Vaccinium arctostaphylos (0.43), Rubus idaeus subsp. idaeus (0.41), Fragaria vesca (0.36) and Hedera helix $(0.34$, Table 2).

The FIC values in the study varies between 0.40 to 0.85 . Rheumatism had the highest FIC value 0.85 with 15 usereports for 3 plant taxa. The plant taxa accountable for the high consensus (0.34) was Hedera helix out of the 58 reported cases. The taxa reported for rheumatism are Thymus vulgaris, Ranunculus cappadocicus and Urtica dioica subsp. dioica. These are followed by cold and flu (0.82) and gynecological diseases (0.71). The lowest FIC values are for hemorrhoid (0.50) and stomach ailments (0.40, Table 3). Gürdal \& Kültür (2013) were determined that the rheumatism had has the highest FIC (0.72) value in their study, similar results were found about the FIC $(0.85)$ value of rheumatism in the present study. Tetik et al. (2013) examined the disease in 10 categories, and they were found that the FIC values range between 0.27 and 0.72 . In addition, their results showed that rheumatism had has the second highest FIC value (0.65).

Ethnomedicinal results showed that the most commonly used parts of plants included leaves, flowers and aboveground, respectively (Figure 2).

The antioxidant, antibacterial and antimicrobial etc. properties of 74 plant taxa, which were identified in the research area, were investigated. Because the pharmacological properties of these plant taxa did not know how the effected on the prepared extract, the contents of the pharmacological activity of these plant taxa have been tried to present with this research according to the previous studies. Within the scope of the study, determined 42 plant taxa had have pharmacological importance with a large percentage as $56.75 \%$ (Table 4).

The numerical distribution of pharmacological properties of these plant taxa are antioxidant (51), antimicrobial (34), antibacterial (19), antitumor (9), antiviral (2), anxiolytic (1), antidepressant (1), anthelmintic (1), antimalarial (2), antidiabetic (2), anti-inflammatory (3), analgesic (2), tumor-inhibitory (1), antiallergic (1), antihepatotoxic (1), antiulcer (3), anticandidal (1), antifungal (3), anticancer (1), antibiotic (1), anticariogenic (1), antigenotoxic (1), antirheumatic (1), antipruritic (1) and antihypertensive (1). It was seen that antioxidant, antimicrobial, antibacterial and antitumor properties had the highest value than other pharmacological properties (Figure 3).

Some plant taxa (21) have neither pharmacological activity nor ethnomedicinal usage. These plant taxa are Aegonychon purpurocaeruleum, Dorycnium graecum, Gymnocarpium dryopteris, Gypsophila tenuifolia, Lathyrus laxiflorus subsp. laxiflorus, Linum austriacum subsp. austriacum, Lythrum maritimum, Oxytropis pilosa, Parentucellia latifolia subsp. latifolia, Pilosella cymosa, Polygala major, Potentilla kotschyana, Saxifraga rotundifolia subsp. rotundifolia, Securigera orientalis subsp. orientalis, Symphytum ibericum, Trifolium aureum subsp. aureum, Tripleurospermum fissurale, Veronica anagalis-aquatica, Veronica filiformis, Veronica multifida, Vicia peregrina. 
Table 2. The Ethnomedicinal plants used by local individuals, in Zorlu Village

\begin{tabular}{|c|c|c|c|c|c|c|c|c|}
\hline Family & Botanical name & Local names & Plant parts & Preparations & $\begin{array}{l}\text { Utilization } \\
\text { method }\end{array}$ & $\begin{array}{l}\text { Ethnomedicinal } \\
\text { Usage }\end{array}$ & $\begin{array}{l}\text { Herbarium } \\
\text { No (KATO) }\end{array}$ & UV \\
\hline Araliaceae & Hedera helix $\mathrm{L}$. & Duvar sarmaşiği & Leaves & Infusion & Drinking & $\begin{array}{l}\text { Rheumatism and } \\
\text { cold }\end{array}$ & 16717 & 0.34 \\
\hline Aristolachiaceae & $\begin{array}{l}\text { Aristolochia pontica } \\
\text { Lam. }\end{array}$ & Loğusa otu & Roots & Decoction & Drinking & $\begin{array}{l}\text { Skin diseases and } \\
\text { stomach ailments, } \\
\text { gynecological } \\
\text { disorders }\end{array}$ & 16747 & 0.29 \\
\hline Asteraceae & Bellis perennis L. & Papatya & $\begin{array}{l}\text { Flowers and } \\
\text { leaves }\end{array}$ & Infusion & Drinking & Cold & 16737 & 0.68 \\
\hline Asteraceae & Cichorium intybus $\mathrm{L}$. & Mavi hindiba & $\begin{array}{l}\text { Above } \\
\text { ground }\end{array}$ & $\begin{array}{l}\text { The above ground } \\
\text { are crushed }\end{array}$ & Compress & Wound & 16716 & 0.08 \\
\hline Asteraceae & $\begin{array}{l}\text { Senecio vernalis } \\
\text { Waldst. \& Kit. }\end{array}$ & Kanarya otu & $\begin{array}{l}\text { Above } \\
\text { ground }\end{array}$ & $\begin{array}{l}\text { The above ground } \\
\text { are crushed }\end{array}$ & Compress & Wound & 16738 & 0.10 \\
\hline Boraginaceae & $\begin{array}{l}\text { Buglossoides arvensis L. } \\
\text { subsp. sibthorpiana R. } \\
\text { Fern }\end{array}$ & Taşkesen & Leaves & Infusion & Drinking & Diuretic diseases & 16746 & 0.06 \\
\hline Boraginaceae & $\begin{array}{l}\text { Echium vulgare subsp. } \\
\text { vulgare } \mathrm{L} \text {. }\end{array}$ & Engerek otu & $\begin{array}{l}\text { Leaves and } \\
\text { flowers }\end{array}$ & Infusion & Drinking & Diuretic diseases & 16719 & 0.18 \\
\hline Boraginaceae & $\begin{array}{l}\text { Onosma sericeum } \\
\text { Willd. }\end{array}$ & Emzik otu & Roots & Decoction & Externally & Hemorrhoid & 16743 & 0.22 \\
\hline Brassicaceae & $\begin{array}{l}\text { Alyssum murale Walds. } \\
\text { \& Kit. subsp. murale } \\
\text { var. murale }\end{array}$ & Kuduz otu & Flowers & Infusion & Drinking & Urinary disorders & 16740 & 0.10 \\
\hline Brassicaceae & Cardamine hirsuta L. & Aci tere & $\begin{array}{l}\text { Above } \\
\text { ground }\end{array}$ & $\begin{array}{l}\text { Decoction and } \\
\text { cooking }\end{array}$ & $\begin{array}{l}\text { Drinking and } \\
\text { eating }\end{array}$ & Strangury & 16708 & 0.43 \\
\hline Caprifoliaceae & Sambucus ebulus L. & Mürver & Leaves & $\begin{array}{l}\text { The leaves are } \\
\text { crushed }\end{array}$ & Compress & Hemorrhoid & 16721 & 0.10 \\
\hline Caryophyllaceae & Stellaria media (L.) Vill. & Kuşotu & Leaves & Decoction & Drinking & Cough & 16761 & 0.15 \\
\hline Cistaceae & Cistus creticus L. & Pembe laden & $\begin{array}{l}\text { Leaves and } \\
\text { flowers }\end{array}$ & Infusion & Drinking & Constipation & 16707 & 0.20 \\
\hline Cistaceae & Cistus salviifolius L. & Beyaz laden & $\begin{array}{l}\text { Leaves and } \\
\text { flowers }\end{array}$ & Infusion & Drinking & $\begin{array}{l}\text { Gynecological } \\
\text { disorders }\end{array}$ & 16715 & 0.13 \\
\hline
\end{tabular}


Ethnobotany Research and Applications

\begin{tabular}{|c|c|c|c|c|c|c|c|c|}
\hline Convolvulaceae & $\begin{array}{l}\text { Convolvulus } \\
\text { cantabricus L. }\end{array}$ & Sarmaşik & $\begin{array}{l}\text { Above } \\
\text { groung }\end{array}$ & Infusion & Drinking & Stomach ailments & 16763 & 0.12 \\
\hline Crassulaceae & Sedum hispanicum L. & Damkoruğu & Leaves & $\begin{array}{l}\text { The leaves are } \\
\text { crushed }\end{array}$ & Compress & Wound & 16724 & 0.06 \\
\hline Dipsacaceae & $\begin{array}{l}\text { Scabiosa columbaria } \\
\text { L.subsp. columbaria var. } \\
\text { columbaria }\end{array}$ & Uyuz otu & Roots & Infusion & Drinking & $\begin{array}{l}\text { Constipation and } \\
\text { diuretic diseases }\end{array}$ & 16762 & 0.12 \\
\hline Ericaceae & $\begin{array}{l}\text { Rhododendron luteum } \\
\text { Sweet }\end{array}$ & Zifin & Leaves & $\begin{array}{l}\text { The leaves are } \\
\text { crushed }\end{array}$ & Compress & Foot infections & 16713 & 0.22 \\
\hline Ericaceae & $\begin{array}{l}\text { Vaccinium } \\
\text { arctostaphylos L. }\end{array}$ & Ayi üzümü & $\begin{array}{l}\text { Leaves, } \\
\text { flowers and } \\
\text { fruits }\end{array}$ & $\begin{array}{l}\text { Infusion, jam and } \\
\text { syrup }\end{array}$ & $\begin{array}{l}\text { Drinking and } \\
\text { eating }\end{array}$ & Kidney diseases & 16718 & 0.43 \\
\hline Guttiferae & Hypericum orientale $\mathrm{L}$. & Kantaron & Flowers & $\begin{array}{l}\text { Olive oil mixed } \\
\text { with flowers }\end{array}$ & Compress & $\begin{array}{l}\text { Hemorrhoid, } \\
\text { gynecological } \\
\text { disorders }\end{array}$ & 16774 & 0.05 \\
\hline Juglandaceae & Juglans regia L. & Ceviz & Seeds & Fresh & Eating & Cholesterol & 16744 & 0.10 \\
\hline Lamiaceae & $\begin{array}{l}\text { Stachys annua (L.) L. } \\
\text { subsp. annua var. } \\
\text { annua }\end{array}$ & Dağ çayi & $\begin{array}{l}\text { Above } \\
\text { ground }\end{array}$ & Infusion & Drinking & Cough & 16731 & 0.05 \\
\hline Lamiaceae & $\begin{array}{l}\text { Teucrium flavum L. } \\
\text { ssubsp. hellenicum } \\
\text { Rech. f. }\end{array}$ & Mayasil otu & Leaves & Infusion & Drinking & Diabetes & 16767 & 0.10 \\
\hline Lamiaceae & $\begin{array}{l}\text { Teucrium polium L. } \\
\text { subsp. polium }\end{array}$ & Mayasil otu & Leaves & Infusion & Drinking & Hemorrhoid & 16750 & 0.10 \\
\hline Lamiaceae & $\begin{array}{l}\text { Thymus nummularius } \\
\text { M. Bieb. }\end{array}$ & Kekik & $\begin{array}{l}\text { Leaves and } \\
\text { flowers }\end{array}$ & Infusion & Drinking & Stomach ailments & 16764 & 0.18 \\
\hline Lamiaceae & $\begin{array}{l}\text { Thymus praecox Opitz } \\
\text { subsp. grossheimii } \\
\text { (Ronniger) Jalas }\end{array}$ & Yayla kekiği & $\begin{array}{l}\text { Above } \\
\text { ground }\end{array}$ & Infusion & Drinking & Stomach ailments & 16726 & 0.17 \\
\hline Lamiaceae & $\begin{array}{l}\text { Thymus praecox subsp. } \\
\text { skorpilii var. skorpilii }\end{array}$ & Yayla Kekiği & $\begin{array}{l}\text { Above } \\
\text { ground }\end{array}$ & Infusion & Drinking & Stomach ailments & 16729 & 0.17 \\
\hline Lamiaceae & Thymus vulgaris $\mathrm{L}$. & Kekik & $\begin{array}{l}\text { Leaves and } \\
\text { flowers }\end{array}$ & Infusion & Drinking & $\begin{array}{l}\text { Rheumatism and } \\
\text { stomach ailments }\end{array}$ & 16736 & 0.22 \\
\hline Leguminosae & $\begin{array}{l}\text { Astragalus caucasicus } \\
\text { Pall. }\end{array}$ & Geven & Roots & Decoction & Drinking & Diabetes & 16711 & 0.10 \\
\hline
\end{tabular}


Ethnobotany Research and Applications

\begin{tabular}{|c|c|c|c|c|c|c|c|c|}
\hline Leguminosae & Genista tinctoria L. & Katir tirnaği & $\begin{array}{l}\text { Above } \\
\text { ground }\end{array}$ & Infusion & Drinking & Urinary disorders & 16757 & 0.15 \\
\hline Leguminosae & $\begin{array}{l}\text { Trifolium pratense } \mathrm{L} \text {. } \\
\text { var. pratense }\end{array}$ & Yonca & $\begin{array}{l}\text { Above } \\
\text { ground }\end{array}$ & Decoction & Drinking & $\begin{array}{l}\text { Sore throat, } \\
\text { diarrhea }\end{array}$ & 16723 & 0.29 \\
\hline Papaveraceae & $\begin{array}{l}\text { Papaver lateritium } \mathrm{C} . \\
\text { Koch subsp. lateritium }\end{array}$ & Gelincik & Leaves & Decoction & Drinking & Cough & 16712 & 0.13 \\
\hline Plantaginaceae & $\begin{array}{l}\text { Plantago major L. } \\
\text { subsp. major }\end{array}$ & $\begin{array}{l}\text { Sinir otu, Damar } \\
\text { otu }\end{array}$ & Leaves & $\begin{array}{l}\text { The leaves are } \\
\text { crushed }\end{array}$ & Compress & Inflamed wounds & 16710 & 0.29 \\
\hline Primulaceae & $\begin{array}{l}\text { Anagallis arvensis L. var. } \\
\text { arvensis }\end{array}$ & $\begin{array}{l}\text { Farekulaği, } \\
\text { Siçankulaği }\end{array}$ & $\begin{array}{l}\text { Above } \\
\text { ground }\end{array}$ & Infusion & Externally & $\begin{array}{l}\text { Wounds and } \\
\text { pimples }\end{array}$ & 16751 & 0.25 \\
\hline Primulaceae & $\begin{array}{l}\text { Primula acaulis } \mathrm{L} \text {. } \\
\text { subsp. rubra }\end{array}$ & Çuha çiçeği & $\begin{array}{l}\text { Leaves and } \\
\text { flowers }\end{array}$ & Infusion & Drinking & Cough & 16725 & 0.22 \\
\hline Ranunculaceae & $\begin{array}{l}\text { Ranunculus } \\
\text { cappadocicus Willd. }\end{array}$ & Su düğün çiçeği & Flowers & $\begin{array}{l}\text { The flowers are } \\
\text { crushed }\end{array}$ & Compress & Rheumatism & 16775 & 0.22 \\
\hline Resedaceae & $\begin{array}{l}\text { Reseda lutea L. var. } \\
\text { lutea }\end{array}$ & Kuzu otu & Young leaves & Fresh & Eating & Stomach ailments & 16756 & 0.18 \\
\hline Rosaceae & Fragaria vesca L. & Dağ çileği & Fruits & Jam and syrup & $\begin{array}{l}\text { Drinking and } \\
\text { eating }\end{array}$ & $\begin{array}{l}\text { Renal diseases } \\
\text { and diuretic } \\
\text { diseases }\end{array}$ & 16709 & 0.36 \\
\hline Rosaceae & Potentilla recta L. & $\begin{array}{l}\text { Parmak otu, sari } \\
\text { ot }\end{array}$ & Flowers & $\begin{array}{l}\text { The flowers are } \\
\text { crushed }\end{array}$ & Compress & Wound & 16732 & 0.12 \\
\hline Rosaceae & $\begin{array}{l}\text { Rubus idaeus L. subsp. } \\
\text { idaeus }\end{array}$ & Böğürtlen & $\begin{array}{l}\text { Fruits and } \\
\text { leaves }\end{array}$ & $\begin{array}{l}\text { Fresh and } \\
\text { decoction }\end{array}$ & $\begin{array}{l}\text { Drinking and } \\
\text { eating }\end{array}$ & Diuretic diseases & 16706 & 0.41 \\
\hline Urticaceae & $\begin{array}{l}\text { Urtica dioica L. subsp. } \\
\text { dioica }\end{array}$ & Isirgan, Cincar & $\begin{array}{l}\text { Leaves and } \\
\text { above } \\
\text { ground }\end{array}$ & $\begin{array}{l}\text { Decoction, } \\
\text { cooking and the } \\
\text { above ground are } \\
\text { crushed }\end{array}$ & $\begin{array}{l}\text { Drinking, eating } \\
\text { and compresses }\end{array}$ & Rheumatism & 16720 & 0.25 \\
\hline
\end{tabular}


Table 3. Factor Informant Consensus (FIC) for each disease

\begin{tabular}{|l|l|l|l|}
\hline Ailment categories & $\begin{array}{l}\text { Number of } \\
\text { use report } \\
\text { (Nur) }\end{array}$ & $\begin{array}{l}\text { Number of } \\
\text { taxa } \\
\text { (Nt) }\end{array}$ & FIC \\
\hline Rheumatism & 15 & 3 & 0.85 \\
\hline Cold and flu & 35 & 7 & 0.82 \\
\hline Gynecological disorders & 8 & 3 & 0.71 \\
\hline Diabetes, Cholesterol & 6 & 3 & 0.60 \\
\hline Skin diseases & 17 & 8 & 0.56 \\
\hline Kidney diseases & 25 & 12 & 0.54 \\
\hline Hemorrhoid & 7 & 4 & 0.50 \\
\hline Stomach ailments & 11 & 7 & 0.40 \\
\hline
\end{tabular}

\section{Plant parts}

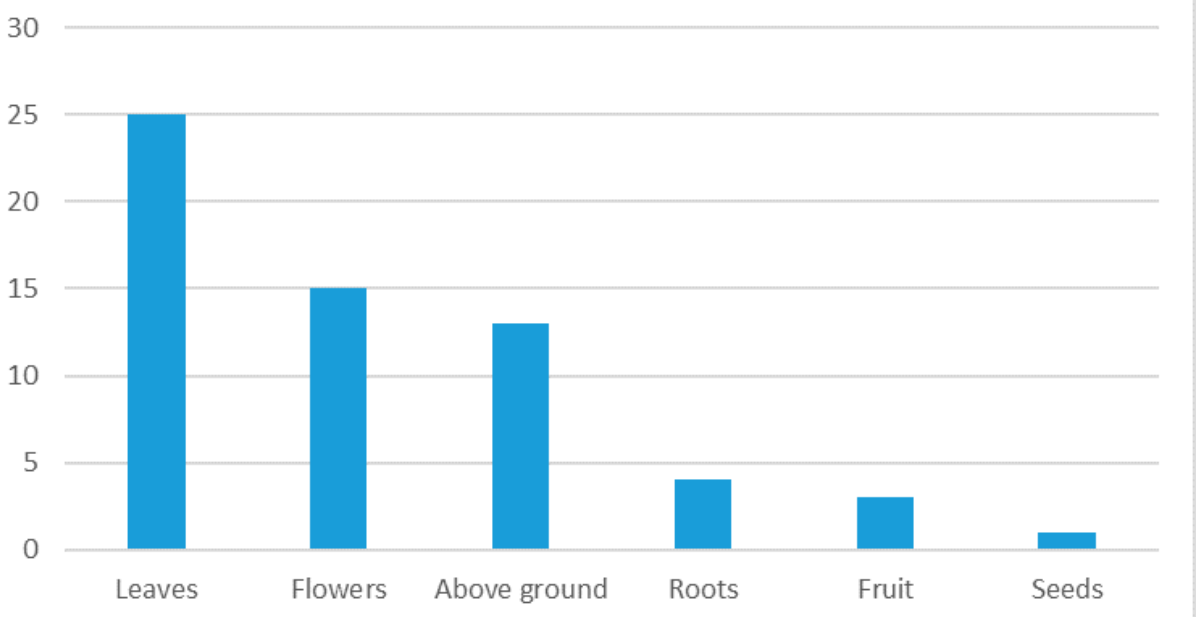

Figure 2. The highest number of usage plant parts

Table 4. Pharmacological properties of the plant taxa

\begin{tabular}{|l|l|l|l|l|}
\hline Family & Botanical name & $\begin{array}{l}\text { Turkish and } \\
\text { *Local name }\end{array}$ & Pharmacological activity & $\begin{array}{l}\text { Herbarium } \\
\text { no (KATO) }\end{array}$ \\
\hline Adoxaceae & Sambucus ebulus & Mürver otu & $\begin{array}{l}\text { Antioxidant and Antimicrobial } \\
\text { activities (Ebrahimzadeh et al. 2009, } \\
\text { Salehzadeh et al. 2014) }\end{array}$ & 16721 \\
\hline Araliaceae & Hedera helix & Duvar sarmaşiği & $\begin{array}{l}\text { Antimicrobial activity (Orhan et al. } \\
\text { 2012) }\end{array}$ & 16717 \\
\hline Aristolachiaceae & $\begin{array}{l}\text { Aristolochia } \\
\text { pontica }\end{array}$ & $\begin{array}{l}\text { Gangirdak } \\
\text { *Loğusa otu }\end{array}$ & $\begin{array}{l}\text { Antioxidant and Antimicrobial } \\
\text { activities (Chawla et al. 2013) }\end{array}$ & 16747 \\
\hline Asteraceae & Achillea biserrata & $\begin{array}{l}\text { Aksirikotu } \\
\text { *Civan perçemi }\end{array}$ & $\begin{array}{l}\text { Antioxidant and Antimicrobial } \\
\text { activities (Serdar et al. 2015, Azaz et } \\
\text { al. 2009) }\end{array}$ & 16734 \\
\hline Asteraceae & Bellis perennis & $\begin{array}{l}\text { Koyungözü } \\
\text { *Papatya }\end{array}$ & $\begin{array}{l}\text { Antioxidant, Anxiolytic, Antitumor } \\
\text { and Antidepressant effects (Siatka } \\
\text { \& Kašparová 2010, Marques et al. } \\
\text { 2012, Pehlivan Karakas et al. 2014) }\end{array}$ & 16737 \\
\hline Asteraceae & Cichorium intybus & Hindiba & $\begin{array}{l}\text { Antimicrobial, Anthelmintic, } \\
\text { Antimalarial, Antidiabetic, Anti- } \\
\text { Inflammatory, Analgesic, } \\
\text { Antioxidant, Tumor-Inhibitory, } \\
\text { Antiallergic and Antihepatotoxic } \\
\text { activities (Street et al. 2013, Ahmed } \\
\text { et al. 2003) }\end{array}$ & 16716 \\
\hline Asteraceae & Sentimicrobial activity (Okach et al. \\
2013) & 16738 \\
\hline
\end{tabular}




\begin{tabular}{|c|c|c|c|c|}
\hline Boraginaceae & $\begin{array}{l}\text { Buglossoides } \\
\text { arvensis subsp. } \\
\text { sibthorpiana }\end{array}$ & Tarla taşkeseni & Antioxidant activity (Tetens 2015) & 16746 \\
\hline Boraginaceae & $\begin{array}{l}\text { Echium vulgare } \\
\text { subsp. vulgare }\end{array}$ & Engerek otu & $\begin{array}{l}\text { Antioxidant, Antibacterial and } \\
\text { Antitumor activities (Nićiforović et } \\
\text { al. 2010, Karakaş et al. 2012) }\end{array}$ & 16719 \\
\hline Caryophyllaceae & $\begin{array}{l}\text { Agrostemma } \\
\text { githago }\end{array}$ & $\begin{array}{l}\text { Buğday } \\
\text { karamuğu }\end{array}$ & $\begin{array}{l}\text { Antibacterial activity (Wagner et al. } \\
\text { 2017) }\end{array}$ & 16739 \\
\hline Caryophyllaceae & Stellaria media & Kuşotu & $\begin{array}{l}\text { Antirheumatic, Anti-inflammatory } \\
\text { and Antipruritic activities (Chandra } \\
\text { \& Rawat 2015) }\end{array}$ & 16761 \\
\hline Cistaceae & Cistus salviifolius & $\begin{array}{l}\text { Kartli } \\
\text { *Beyaz laden }\end{array}$ & $\begin{array}{l}\text { Antioxidant, Antimicrobial and } \\
\text { Antibacterial activities (Sayah et al. } \\
\text { 2017, Tomás-Menor et al. 2013) }\end{array}$ & 16715 \\
\hline Dipsacaceae & $\begin{array}{l}\text { Scabiosa } \\
\text { columbaria subsp. } \\
\text { columbaria var. } \\
\text { columbaria }\end{array}$ & Uyuzotu & $\begin{array}{l}\text { Antimicrobial activity (Moteetee \& } \\
\text { Kose 2017) }\end{array}$ & 16762 \\
\hline Ericaceae & $\begin{array}{l}\text { Vaccinium } \\
\text { arctostaphylos }\end{array}$ & $\begin{array}{l}\text { Likarpa } \\
\text { ^Ayi üzümü }\end{array}$ & $\begin{array}{l}\text { Antioxidant, Antimicrobial and } \\
\text { Antihypertensive } \\
\text { Activities (Güder et al. 2014, } \\
\text { Mahboubi et al. 2013, Khalili et al. } \\
\text { 2011) }\end{array}$ & 16718 \\
\hline Fabaceae & $\begin{array}{l}\text { Astragalus } \\
\text { caucasicus }\end{array}$ & Kaf geveni & $\begin{array}{l}\text { Antimicrobial and Antiviral activities } \\
\text { (Li et al. 2014) }\end{array}$ & 16711 \\
\hline Fabaceae & Genista tinctoria & $\begin{array}{l}\text { Boyaci } \\
\text { katirtirnaği }\end{array}$ & $\begin{array}{l}\text { Antiulcer and Antioxidant activities } \\
\text { (Kumari \& Prasad 2013, Antal et al. } \\
\text { 2010) }\end{array}$ & 16757 \\
\hline Fabaceae & Medicago lupulina & Bitçikotu & $\begin{array}{l}\text { Antibacterial activity (Anonymous } \\
\text { 2016; Ergül Bozkurt \& Terzioğlu } \\
\text { 2017) }\end{array}$ & 16722 \\
\hline Fabaceae & Trifolium dubium & Tatli yonca & $\begin{array}{l}\text { Antibacterial and Antifungal } \\
\text { activities (Ali-Shtayeh et al. 2015) }\end{array}$ & 16759 \\
\hline Fabaceae & $\begin{array}{l}\text { Trifolium pratense } \\
\text { var. pratense }\end{array}$ & Çayir üçgülü & $\begin{array}{l}\text { Antibacterial activity (Dobrucka \& } \\
\text { Długaszewska 2016) }\end{array}$ & 16723 \\
\hline Geraniaceae & $\begin{array}{l}\text { Geranium } \\
\text { columbinum }\end{array}$ & $\begin{array}{l}\text { Güvercin itiri } \\
\text { *Uzun sapli } \\
\text { Turnagagasi }\end{array}$ & $\begin{array}{l}\text { Antimicrobial activity (Radulovic et } \\
\text { al. 2011) }\end{array}$ & 16749 \\
\hline Geraniaceae & Geranium molle & $\begin{array}{l}\text { Yumuşak itir } \\
\text { *Tüylü } \\
\text { Turnagagasi }\end{array}$ & $\begin{array}{l}\text { Antioxidant activity (Graça et al. } \\
\text { 2016) }\end{array}$ & \\
\hline Geraniaceae & $\begin{array}{l}\text { Geranium } \\
\text { purpureum }\end{array}$ & $\begin{array}{l}\text { Ebedön } \\
\text { *Turnagagasi }\end{array}$ & $\begin{array}{l}\text { Antioxidant and Antimicrobial } \\
\text { activities (Proestos et al. 2006, } \\
\text { Cardoso \& Matos 2013) }\end{array}$ & 16714 \\
\hline Guttiferae & $\begin{array}{l}\text { Hypericum } \\
\text { orientale }\end{array}$ & $\begin{array}{l}\text { Sandik çiçeği } \\
\text { *Sari kantaron }\end{array}$ & $\begin{array}{l}\text { Antidepressant effects (Medina et } \\
\text { al. 2006) }\end{array}$ & 16774 \\
\hline Juglandaceae & Juglans regia & $\begin{array}{l}\text { Ceviz } \\
\text { *Kakali }\end{array}$ & $\begin{array}{l}\text { Anticandidal, Antifungal, Antitumor, } \\
\text { Antioxidant and Antibacterial } \\
\text { activities (Noumi et al. 2010, Noumi } \\
\text { et al. 2011, Santos et al. 2013, } \\
\text { Rather et al. 2012) }\end{array}$ & 16744 \\
\hline Lamiaceae & Acinos arvensis & *Tarla nanesi & $\begin{array}{l}\text { Antioxidant and Antimicrobial } \\
\text { activities (Jovanovic et al. 2005, } \\
\text { Kaya et al. 1999) }\end{array}$ & 16730 \\
\hline Lamiaceae & Ajuga reptans & $\begin{array}{l}\text { Meryemsaçi } \\
\text { ^Dağ } \\
\text { mayasilotu }\end{array}$ & $\begin{array}{l}\text { Strong antitumor activity (Yildirim } \\
\text { et al. 2012) }\end{array}$ & 16735 \\
\hline Lamiaceae & $\begin{array}{l}\text { Stachys annua } \\
\text { subsp. annua var. } \\
\text { annua }\end{array}$ & $\begin{array}{l}\text { Haciosmanotu } \\
\text { *Dağ çayi }\end{array}$ & $\begin{array}{l}\text { Antibacterial and Antimicrobial } \\
\text { activities (Yildirim et al. 2013) }\end{array}$ & 16731 \\
\hline Lamiaceae & $\begin{array}{l}\text { Teucrium polium } \\
\text { subsp. polium }\end{array}$ & $\begin{array}{l}\text { Aciyavşan } \\
\text { *Mayasil otu }\end{array}$ & $\begin{array}{l}\text { Antioxidant and Antimicrobial } \\
\text { activities (Khaled-Khodja et al. } \\
\text { 2014, Sarac \& Ugur 2007) }\end{array}$ & 16750 \\
\hline
\end{tabular}




\begin{tabular}{|c|c|c|c|c|}
\hline Lamiaceae & $\begin{array}{l}\text { Thymus } \\
\text { nummularius }\end{array}$ & Limon kekiği & $\begin{array}{l}\text { Antioxidant activity (Ertas et al. } \\
\text { 2015) }\end{array}$ & 16764 \\
\hline Lamiaceae & $\begin{array}{l}\text { Thymus praecox } \\
\text { subsp. skorpilii } \\
\text { var. skorpilii }\end{array}$ & Yayla kekiği & $\begin{array}{l}\text { Antioxidant activity (Ozen et al. } \\
\text { 2011) }\end{array}$ & 16729 \\
\hline Lamiaceae & Thymus vulgaris & Kekik & $\begin{array}{l}\text { Antibacterial activity (Dorman } \& \\
\text { Deans 2000) }\end{array}$ & 16736 \\
\hline Plantaginaceae & $\begin{array}{l}\text { Plantago major } \\
\text { subsp. major }\end{array}$ & $\begin{array}{l}\text { Sinirotu } \\
\text { *Damarli ot, } \\
\text { *Balazağva }\end{array}$ & $\begin{array}{l}\text { Antiulcerogenic, Anticancer, } \\
\text { Antibiotic, Antifungal, } \\
\text { Antigiardiasic, Antimalarial, } \\
\text { Antiviral, Antioxidant activities } \\
\text { (Samuelsen 2000, Stanisavljević et } \\
\text { al. 2008) }\end{array}$ & 16710 \\
\hline Primulaceae & $\begin{array}{l}\text { Anagallis arvensis } \\
\text { var. arvensis }\end{array}$ & $\begin{array}{l}\text { Farekulaği } \\
\text { *Siçankulaği }\end{array}$ & $\begin{array}{l}\text { Antioxidant and Antibacterial } \\
\text { activities (Tawaha et al. 2007, Taye } \\
\text { et al. 2011) }\end{array}$ & 16751 \\
\hline Primulaceae & $\begin{array}{l}\text { Primula acaulis } \\
\text { subsp. rubra }\end{array}$ & $\begin{array}{l}\text { Evvelbahar } \\
\text { çiçeği } \\
\text { *Çuha çiçeği }\end{array}$ & $\begin{array}{l}\text { Antioxidant and Antigenotoxic } \\
\text { activities (Ozkan et al. 2017) }\end{array}$ & 16725 \\
\hline Resedaceae & $\begin{array}{l}\text { Reseda lutea var. } \\
\text { lutea }\end{array}$ & $\begin{array}{l}\text { Muhabbet } \\
\text { çiçeği } \\
{ }^{\star} \text { Kuzu otu }\end{array}$ & $\begin{array}{l}\text { Antimicrobial and Antibacterial } \\
\text { activities (Jafari-Sales et al. 2017) }\end{array}$ & 16756 \\
\hline Rosaceae & Fragaria vesca & Dağ çileği & $\begin{array}{l}\text { Antioxidant activity (Žugić et al. } \\
\text { 2014) }\end{array}$ & 16709 \\
\hline Rosaceae & $\begin{array}{l}\text { Potentilla } \\
\text { argentea }\end{array}$ & $\begin{array}{l}\text { Gümüş } \\
\text { parmakotu }\end{array}$ & Antioxidant activity (Antal 2010) & 16748 \\
\hline Rosaceae & $\begin{array}{l}\text { Potentilla crantzii } \\
\text { var. crantzii }\end{array}$ & Beşparmakotu & $\begin{array}{l}\text { Antimicrobial activity (Tomczyk et } \\
\text { al. 2010) }\end{array}$ & 16752 \\
\hline Rosaceae & Potentilla recta & $\begin{array}{l}\text { Su parmakotu } \\
\text { *Sari ot }\end{array}$ & $\begin{array}{l}\text { Antioxidant, Anti-inflammatory and } \\
\text { Anticariogenic activities (Tomczyk } \\
\text { et al. 2011, Bazylko et al. 2013) }\end{array}$ & 16732 \\
\hline Rosaceae & $\begin{array}{l}\text { Potentilla } \\
\text { thuringiaca }\end{array}$ & Koç parmakotu & $\begin{array}{l}\text { Antioxidant activity (Grochowski et } \\
\text { al. 2017) }\end{array}$ & 16754 \\
\hline Rosaceae & $\begin{array}{l}\text { Rubus idaeus } \\
\text { subsp. idaeus }\end{array}$ & Ahududu & $\begin{array}{l}\text { Antimicrobial, Antioxidant and } \\
\text { Antibacterial activities (Rauha et al. } \\
\text { 2000, Deighton et al. 2000, } \\
\text { Cavanagh et al. 2003) }\end{array}$ & 16706 \\
\hline Urticaceae & $\begin{array}{l}\text { Urtica dioica } \\
\text { subsp. dioica. }\end{array}$ & $\begin{array}{l}\text { Isirgan } \\
{ }^{*} \text { Cincar }\end{array}$ & $\begin{array}{l}\text { Antioxidant, Antimicrobial } \\
\text { Antiulcer, Analgesic and } \\
\text { Antidiabetic activities (Kukrić et al. } \\
\text { 2012, Gülçin et al. 2004, Bahmani et } \\
\text { al. 2014) }\end{array}$ & 16720 \\
\hline
\end{tabular}

\section{Pharmacological activity}

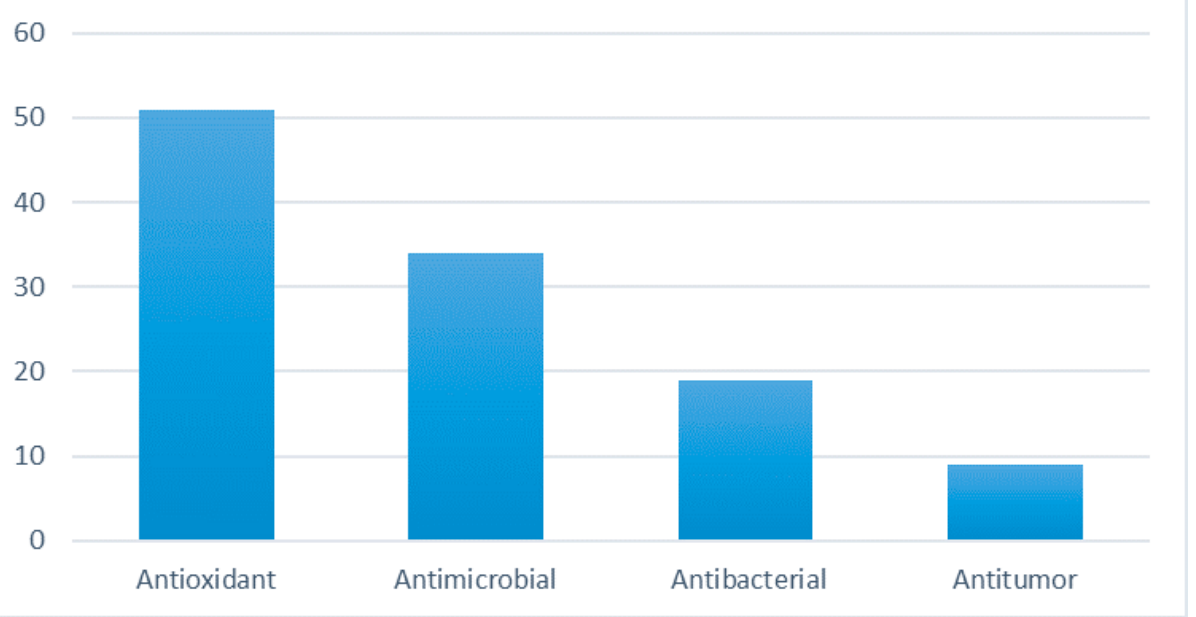

Figure 3. The highest number of Pharmacological properties 
As a result of the study, phytogeographic region and endemism value of the plant taxa were investigated and it was determined that 11 plant taxa had has phytogeographic region and only 1 plant taxon was endemic. Botanical name, phytogeographic region and endemism status of these plant taxa showed in Figure 4.

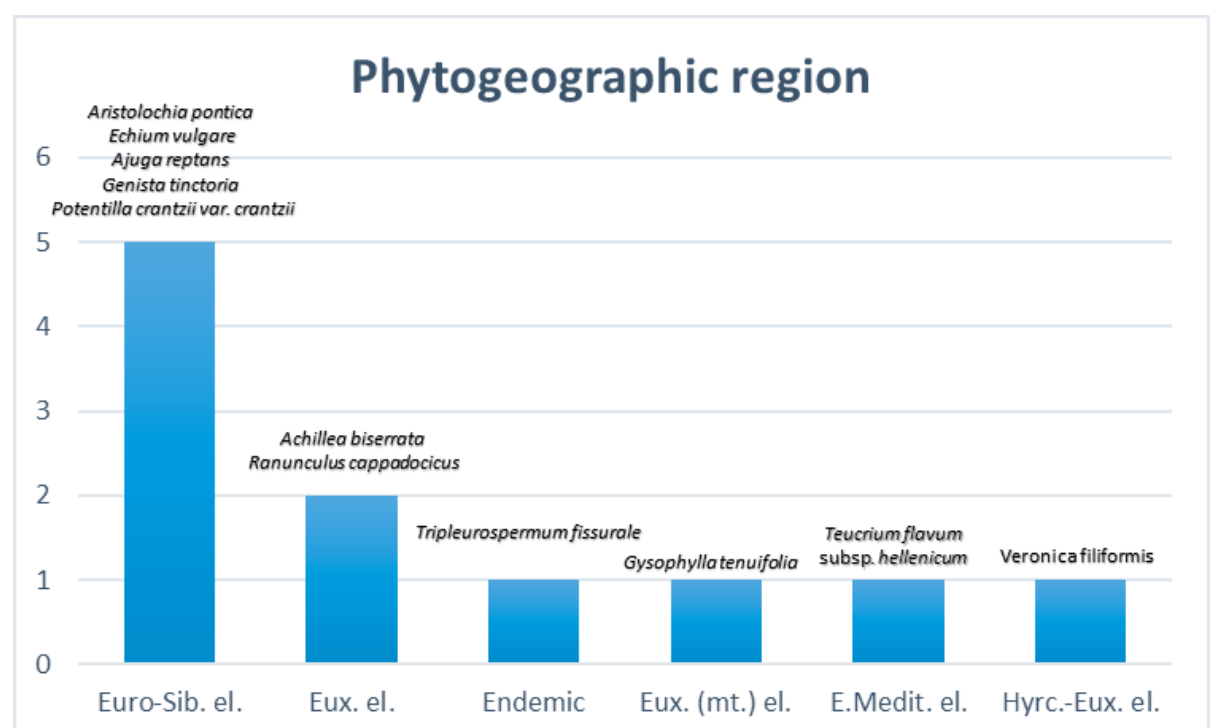

Figure 4. Botanical name, phytogeographic region and endemism status of the plant taxa

At the end of the study, the most common species are found from the families of Fabaceae with 11 taxa, Lamiaceae with 9 taxa, Rosaceae with 7 taxa, Asteraceae with 6 taxa and Boraginaceae with 6 taxa (Figure 5). Similarly, Saraç et al. (2013) found that the largest five families were qualified as follows: Asteraceae family with 14 taxa, Rosaceae family with 11 taxa, Lamiaceae family with 10 taxa, Ericaceae and Fabaceae with 4 taxa in each.

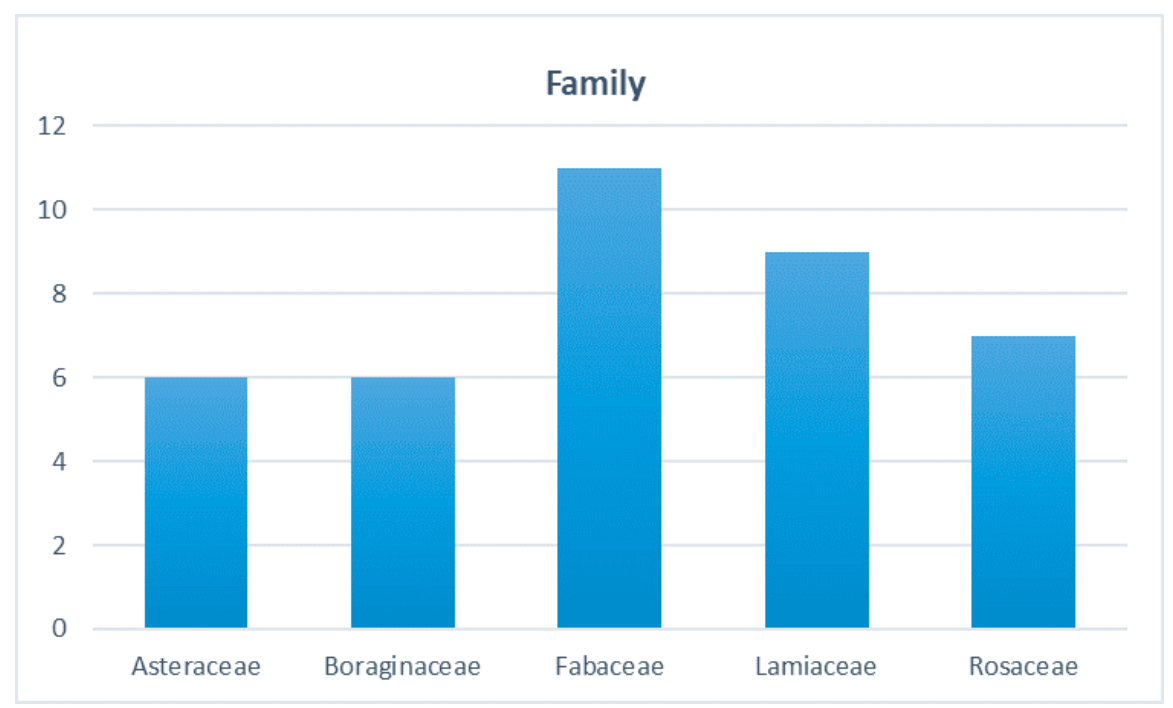

Figure 5. The families of the highest number of plant taxa

Ethnopharmacology involves the investigation of plant taxa use in traditional communities, for that reason the research of this issue is quite important (Bhatia et al. 2014). Because herbal medicines are relatively safer than synthetic drugs (Jagatheeswari 2012). In most developing countries, women are generally reluctant to subject themselves to gynecological examination, particularly when they have no apparent symptoms of disease due to lack of awareness and shyness (Bhatia \& Cleland 1995). Similarly, Shrivastava (2013) determined that the ethnomedicinal plants used for the treatment of gynecological disorders by the tribes of Dindori district. These people do not prefer the doctors, due to lack of awareness and shyness or hesitation. Jan et al. (2020) stated that the indigenous community of district Buner, Pakistan was more sensitive and careful about gynecological diseases. Rahman et al. (2014) investigated the plants, which would be good for gynecological diseases in the region of Dinajbur, Bangladesh and they determined that which parts of the plants had have the healing effects on local people. An ethnobotanical survey of medicinal plants used in the treatment of gynecological disorders was carried 
out by Buragohain (2008) among the rural people in Tinsukia district, Assam, India. As a result of that study, he was suggested that the scientific validation and clinical proof with these folk herbal medicines might lead to potential drugs. Yadav et al. (2006) suggested that documentation of ethnomedicinal data on biological resources will be steps for bioprospecting. Similar results have been obtained in many previous studies about traditional treatment of gynecological diseases (Abu-Irmaileh \& Afifi 2003; Steenkamp 2003; Behera 2006; Vidyasagar \& Prashantkumar 2007; Zhou \& Qu 2009; Chaurasia 2011; Singh \& Mall 2011; Wadankar et al. 2011; Gupta \& Solanki 2013; Das et al. 2015; Ögenler et al. 2018; Balamurugan et al. 2018). Therefore, studies on traditional treatment of gynecological diseases should be increased. And a scientific link should be established between traditional treatment methods and modern medical methods.

\section{Conclusion}

As a result of the study, 74 plant taxa under 30 families were determined. 41 plant taxa $(55.40 \%)$ had ethnomedicinal importance and 42 plant taxa had pharmacological importance with a large percentage as $56.75 \%$.

The results showed that pharmacological properties (antioxidant, antimicrobial, antibacterial etc. activities) play an effective role on the preferred plants (74) extract, which is used by the rural population of Zorlu village in Turkey.

The present study focused on the utilization of plants available with the people of Zorlu Village, they were used the traditional knowledge for the treatment of gynecological disorders. Because of the urgent need for systematic documentation of this knowledge by using scientific tools, I want to share this knowledge. In order to understand the potential of the treatment in traditional medicine on gynecological disorders, there is a need for more studies related to the pharmacological and clinical research.

\section{Declarations}

Ethics approval and consent to participate: All participants provided prior informed consent before any interviews were conducted. And they were informed about the aim of this study.

Consent for publication: Not applicable.

Competing interests: Author declares that there is no conflict of interest.

Funding: This study did not receive any funding.

\section{Acknowledgements}

The author would like to thank her grandmother Pakize ALTUNTAŞ and local individuals of Zorlu Village in Borçka/Artvin (Turkey).

\section{Literature Cited}

Abraham Z. 1981. Glimpses of Indian Ethnobotany. Oxford and Publishing Co., New Delhi: pp. 308-320.

Abu-Irmaileh BE, Afifi FU. 2003. Herbal medicine in Jordan with special emphasis on commonly used herbs. Journal of Ethnopharmacology 89(2-3):193-197.

Ahmed B, Al-Howiriny, TA, Siddiqui AB. 2003. Antihepatotoxic activity of seeds of Cichorium intybus. Journal of Ethnopharmacology 87(2):237-240.

Ali-Shtayeh MS, Jamous RM, Abu Zaitoun SY. 2015. A Comprehensive Science-Based Field Assessment of Bioactive Properties of the Native Plants of Palestine. Journal of Biodiversity Bioprospecting and Development 2(151):23760214.

Anonymous 2016. www.naturalmedicinalherbs.net/herbs/m/medicago-lupulina. Retrieved on 21 November 2016.

Anonymous. 1994. Borçka (Artvin) Camili-Karagöl orman ekosistemlerini koruma ve geliştirme olanaklari araştirmasi. Kirsal Çevre ve Ormancilik Sorunlarini Araştirma Derneği, Ankara.

Anonymous. 2002. Çevre Bakanliği Devlet Meteoroloji İşleri Genel Müdürlüğü Rasat Bilgisi 6936, Ankara.

Antal DS. 2010. Medicinal plants with antioxidant properties from Banat region (Romania): a rich pool for the discovery of multi-target phytochemicals active in free-radical related disorders. Analele Universitatii din OradeaFascicula Biologie 17:1. 
Atal CK, Kapur BM. 1982. Cultivation and utilization of medicinal plants. Jammu-Tawi, India: Regional Research Laboratory, Council of Scientific \& Industrial Research.

Atalay i. 1983. A general survey of the vegetation of north-eastern Anatolia. Ege Coğrafya Dergisi 1(1).

Azaz AD, Arabaci T, Sangun MK. 2009. Essential oil composition and antimicrobial activities of Achillea biserrata M. Bieb. and Achillea salicifolia Besser subsp. salicifolia collected in Turkey. Asian Journal of Chemistry 21(4):31933198.

Bahmani M, Zargaran A, Rafieian-Kopaei M, Saki K. 2014. Ethnobotanical study of medicinal plants used in the management of diabetes mellitus in the Urmia, Northwest Iran. Asian Pacific Journal of Tropical Medicine 7:S348S354.

Balamurugan S, Vijayakumar S, Prabhu S, Yabesh J M. 2018. Traditional plants used for the treatment of gynaecological disorders in Vedaranyam taluk, South India-an ethnomedicinal survey. Journal of Traditional and Complementary Medicine 8(2):308-323.

Bazylko A, Piwowarski JP, Filipek A, Bonarewicz J, Tomczyk M. 2013. In vitro antioxidant and anti-inflammatory activities of extracts from Potentilla recta and its main ellagitannin, agrimoniin. Journal of Ethnopharmacology, 149(1):222-227.

Behera KK. 2006. Plants used for gynecological disorders by tribals of Mayurbhanj district, Orissa, India. Ethnobotanical Leaflets 2006(1),:15.

Bhatia H, Sharma YP, Manhas RK, Kumar K. 2014. Ethnomedicinal plants used by the villagers of district Udhampur. J\&K, India. Journal of Ethnopharmacology 151(2):1005-1018.

Bhatia JC, Cleland J. 1995. Self-reported symptoms of gynecological morbidity and their treatment in south India. Studies in Family Planning 26(4):203-217.

Buragohain J. 2008. Folk medicinal plants used in gynecological disorders in Tinsukia district, Assam, India. Fitoterapia 79(5):388-392.

Camejo-Rodrigues J, Ascensao L, Bonet MÀ, Valles J. 2003. An ethnobotanical study of medicinal and aromatic plants in the Natural Park of "Serra de São Mamede" (Portugal). Journal of Ethnopharmacology 89(2-3):199-209.

Cardoso F, Matos O. 2013. Screening of methanol Portuguese ethnobotanical plant extracts for the antimicrobial activity. Worldwide Research Efforts in the Fighting Against Microbial Pathogen 235.

Cavanagh HM, Hipwell M, Wilkinson JM. 2003. Antibacterial activity of berry fruits used for culinary purposes. Journal of Medicinal Food 6(1):57-61.

Chandra S, Rawat DS. 2015. Medicinal plants of the family Caryophyllaceae: a review of ethno-medicinal uses and pharmacological properties. Integrative Medicine Research 4(3):123-131.

Chaurasia OP. 2011. Herbal formulations from cold desert plants used for gynecological disorders. Ethnobotany Research and Applications 9:59-66.

Chawla P, Chawla A, Shah G, Baghel US, Dhawan RK. 2013. A Review on Pharmacognosy and Biological Activities of Aristolochia. Asian Journal of Research in Biological and Pharmaceutical Sciences 1(2):97-106.

Das DC, Sinha NK, Das M. 2015. The use of medicinal plants for the treatment of gynecological disorders in the eastern parts of India. Indian Journal of Obstetrics and Gynecology 2(1):16-27.

Davis PH. (Ed.) 1965-1985. Flora of Turkey and the East Aegean Islands. Vol. 1-9, Edinburgh University Press, Edinburgh.

Deighton N, Brennan R, Finn C, Davies HV. 2000. Antioxidant properties of domesticated and wild Rubus species, Journal of the Science of Food and Agriculture 80(9):1307-1313.

Dobrucka R, Długaszewska J. 2016. Biosynthesis and antibacterial activity of ZnO nanoparticles using Trifolium pratense flower extract. Saudi Journal of Biological Sciences 23(4):517-523.

Dorman HJD, Deans SG. 2000. Antimicrobial agents from plants: antibacterial activity of plant volatile oils. Journal of Applied Microbiology 88(2):308-316. 
Ebrahimzadeh MA, Nabavi SF, Nabavi SM. 2009. Antioxidant activities of methanol extract of Sambucus ebulus $\mathrm{L}$. flower. Pakistan Journal of Biological Sciences 12(5):447.

Ergül Bozkurt A, Terzioğlu S. 2017. The aromatic-medicinal plant taxa of pure scots pine stands in SürmeneCamburnu (Trabzon). International Journal of Secondary Metabolites 4(3, Special Issue 2):517-529.

Ertas A, Boga M, Yilmaz MA, Yesil Y, Tel G, Temel H, Hasimi N, Gazioglu I, Öztürk M, Ugurlu P. 2015. A detailed study on the chemical and biological profiles of essential oil and methanol extract of Thymus nummularius (Anzer tea): Rosmarinic acid. Industrial Crops and Products 67:336-345.

Farnsworth NR, Soejarto DD. 1991. Global importance of medicinal plants, In: Akerele O, Heywood V, and Synge H (Eds.), The Conservation of Medicinal Plants. Cambridge University Press, Cambridge, 25-51.

Giday M, Asfaw Z, Woldu Z. 2009. Medicinal plants of the Meinit ethnic group of Ethiopia: an ethnobotanical study. Journal of Ethnopharmacology 124(3):513-521.

Graça VC, Barros L, Calhelha RC, Dias MI, Carvalho AM, Santos-Buelga C, Ferreira ICFR, Santos PF. 2016. Chemical characterization and bioactive properties of Geranium molle L. from the plant to the most active extract and its phytochemicals. Food \& Function 7(5):2204-2212.

Grochowski DM, Uysal S, Aktumsek A, Granica S, Zengin G, Ceylan R, Locatelli M, Tomczyk M. 2017. In vitro enzyme inhibitory properties, antioxidant activities, and phytochemical profile of Potentilla thuringiaca. Phytochemistry Letters.

Gupta U, Solanki H. 2013. Herbal folk remedies used in treatment of Gynecological disorders by tribals of Simalwara Region, Dungarpur, Rajasthan. International Journal of Pure and Applied Sciences and Technology 17(1):100.

Güder A, Engin MS, Yolcu M, Gür M. 2014. Effect of processing temperature on the chemical composition and antioxidant activity of Vaccinium arctostaphylos fruit and their jam. Journal of Food Processing and Preservation 38(4):1696-1704.

Gülçin I, Küfrevioğlu Öi, Oktay M, Büyükokuroğlu ME. 2004. Antioxidant, antimicrobial, antiulcer and analgesic activities of nettle (Urtica dioica L.). Journal of Ethnopharmacology 90(2-3):205-215.

Güner A, Akyildirim B, Alkayiş MF, Çingay B, Kanoğlu SS, Özkan AM, Öztekin M, Tuğ GN. 2012. Türkçe Bitki Adlari. in: Güner A, Aslan S, Ekim T, Vural M, Babaç MT. (Eds.), Türkiye Bitkileri Listesi (Damarli Bitkiler), İstanbul, Nezahat Gökyiğit Botanik Bahçesi ve Flora Araştirmalari Derneği Yayini, 1290p.

Gürdal B, Kültür ş. 2013. An ethnobotanical study of medicinal plants in Marmaris (Muğla, Turkey). Journal of Ethnopharmacology 146:113-126.

Hamayun M, Khan A, Khan M. 2003. Common medicinal folk recipes of District Buner, NWFP. Ethnobototanical Leaflets.

Jafari-Sales A, Bagherizadeh Y, Malekzadeh P, Ahmadi B, Bonab FR. 2017. Evaluation of the antimicrobial effects of essential oil of Reseda lutea L. on pathogenic bacteria: Staphylococcus aureus, Staphylococcus epidermidis, and Escherichia coli. Archives of Clinical Microbiology 8(3).

Jagatheeswari D. 2012. A survey of some medicinally important plants in Villupuram District of Tamil Nadu, India. International Journal of Pharmaceutical and Biological Archives 3(4):905-909.

Jan HA, Jan S, Bussmann RW, Ahmad L, Wali S, Ahmad N. 2020. Ethnomedicinal survey of the plants used for gynecological disorders by the indigenous community of District Buner, Pakistan. Ethnobotany Research and Applications 19.

Johns T, Kokwaro JO, Kimanani EK. 1990. Herbal remedies of the Luo of Siaya District, Kenya: establishing quantitative criteria for consensus. Economic Botany 44:369-381.

Jovanovic T, Kitic D, Palic R, Stojanovic G, Ristic M. 2005. Chemical composition and antimicrobial activity of the essential oil of Acinos arvensis (Lam.) Dandy from Serbia, Flavour and Fragrance Journal 20:288-290.

Karakaş FP, Yildirim A, Türker A. 2012. Biological screening of various medicinal plant extracts for antibacterial and antitumor activities. Turkish Journal of Biology 36(6):641-652. 
Kaya A, Başer KHC, Tümen G, Koca F. 1999. The essential oil of Acinos suaveolens (Sm.) G. Don fil. Acinos arvensis (Lam.) Dandy and Acinos rotundifolius Pers. growing wild in Turkey. Flavour and Fragrance Journal 1: 60-64.

Khaled-Khodja N, Boulekbache-Makhlouf L, Madani K. 2014. Phytochemical screening of antioxidant and antibacterial activities of methanolic extracts of some Lamiaceae. Industrial Crops and Products 61:41-48.

Khalili A, Khosravi MB, Nekooeian AA. 2011. The effects of aqueous extract of Vaccinium arctostaphylos leaves on blood pressure in renal hypertensive rats. Iranian red Crescent Medical Journal 13(2):123.

Kolayli S \& Şahin B. 2007. A taxonomic study on the phytoplankton in the littoral zone of Karagöl Lake (BorçkaArtvin/Turkey). Turkish Journal of Fisheries and Aquatic Sciences 7(2).

Krishnaraju AV, Rao TV, Sundararaju D, Vanisree M, Tsay HS, Subbaraju GV. 2005. Assessment of bioactivity of Indian medicinal plants using brine shrimp (Artemia salina) lethality assay. International Journal of Applied Science and Engineering 3(2):125-34.

Kukrić ZZ, Topalić-Trivunović LN, Kukavica BM, Matoš SB, Pavičić SS, Boroja MM, Savić AV. 2012. Characterization of antioxidant and antimicrobial activities of nettle leaves (Urtica dioica L.). Acta Periodica Technologica 43:257-272.

Kumari R, Prasad MNV. 2013. Medicinal plant active compounds produced by UV-B exposure. Sustainable Agriculture Reviews 225-254.

Li X, Qu L, Dong Y, Han L, Liu E, Fang S, Zhang Y, Wang T. 2014. A review of recent research progress on the Astragalus genus. Molecules 19(11):18850-18880.

Mahboubi M, Kazempour N, Taghizadeh M. 2013. In vitro antimicrobial and antioxidant activity of Vaccinium arctostaphylos L. extracts. Journal of Biologically Active Products from Nature 3(4):241-247.

Marques THC, Melo CHSD, Freitas RMD. 2012. In vitro evaluation of antioxidant, anxiolytic, and antidepressant-like effects of the Bellis perennis extract. Revista Brasileira de Farmacognosia, 22(5):1044-1052.

Medina MA, Martinez-Poveda B, Amores-Sanchez MI, Quesada AR. 2006. Hyperforin, more than an antidepressant bioactive compound? Life Science 79:105-111.

Moteetee A, Kose LS. 2017. A review of medicinal plants used by the Basotho for treatment of skin disorders: their phytochemical, antimicrobial, and anti-inflammatory potential. African Journal of Traditional, Complementary and Alternative Medicines (AJTCAM) 14(5):121-137.

Nićiforović N, Mihailović V, Mašković P, Solujić S, Stojković A, Muratspahić DP. 2010. Antioxidant activity of selected plant species; potential new sources of natural antioxidants. Food and Chemical Toxicology 48(11):3125-3130.

Noumi E, Snoussi M, Hajlaoui H, Valentin E, Bakhrouf A. 2010. Antifungal properties of Salvadora persica and Juglans regia L. extracts against oral Candida strains. European Journal of Clinical Microbiology \& Infectious Diseases 29(1):81.

Noumi E, Snoussi M, Trabelsi N, Hajlaoui H, Ksouri R, Valentin E, Bakhrouf A. 2011. Antibacterial, anticandidal and antioxidant activities of Salvadora persica and Juglans regia L. extracts. Journal of Medicinal Plants Research 5(17):4138-4146.

Okach DO, Nyunja ARO, Opande G. 2013. Phytochemical screening of some wild plants from Lamiaceae and their role in traditional medicine in Uriri District-Kenya. International Journal of Herbal Medicine 1(5):135-143.

Orhan DD, Özçelik B, Hoşbaş S, Vural M. 2012. Assessment of antioxidant, antibacterial, antimycobacterial, and antifungal activities of some plants used as folk remedies in Turkey against dermatophytes and yeast-like fungi. Turkish Journal of Biology 36(6):672-686.

Ozen T, Demirtas I, Aksit H. 2011. Determination of antioxidant activities of various extracts and essential oil compositions of Thymus praecox subsp. skorpilii var. skorpilii. Food Chemistry 124(1):58-64.

Ozkan MT, Aliyazicioglu R, Demir S, Misir S, Turan I, Yildirmis S, Aliyazicioglu Y. 2017. Phenolic characterisation and antioxidant activity of Primula vulgaris and its antigenotoxic effect on fibroblast cell, Jundishapur. Journal of Natural Pharmaceutical Products 12(1).

Ögenler O, Ün I, Uzel i. 2018. Medical plants used for treatment of gynecological disorders in Ottomans in the 15th century. Journal of Complementary Medicine 7(2):171-177. 
Pehlivan Karakas F, Şöhretoğlu D, Liptaj T, Štujber M, Ucar Turker A, Marák J, Çaliş i, Yalçin FN. 2014. Isolation of an oleanane-type saponin active from Bellis perennis through antitumor bioassay-guided procedures. Pharmaceutical Biology 52(8):951-955.

Proestos C, Boziaris IS, Nychas GJ, Komaitis M. 2006. Analysis of flavonoids and phenolic acids in Greek aromatic plants: Investigation of their antioxidant capacity and antimicrobial activity. Food Chemistry 95(4: 664-671.

Qureshi R, Bhatti GR, Memon RA. 2010. Ethnomedicinal uses of herbs from northern part of Nara desert, Pakistan. Pakistan Journal of Botany 42(2):839-851.

Radulovic N, Dekic M, Stojanovic-Radic Z, Palic R. 2011. Chemical composition and antimicrobial activity of the essential oils of Geranium columbinum L. and G. lucidum L. (Geraniaceae). Turkish Journal of Chemistry 35(3):499512.

Rahman AHMM, Jahan-E-Gulsan SM, Naderuzzaman ATM. 2014. Ethno-Gynecological Disorders of Folk Medicinal Plants Used by Santhals of Dinajpur District, Bangladesh. Frontiers of Biological \& Life Sciences 2(3):62-66.

Rama Rao N, Henry N. 1996. The Ethnobotany of Eastern Ghats in Andhra Pradesh, India. Botanical Survey of India, Calcutta.

Rather MA, Dar BA, Dar MY, Wani BA, Shah WA, Bhat BA, Ganai BA, Bhat KA, Anand R, Qurishi MA. 2012. Chemical composition, antioxidant, and antibacterial activities of the leaf essential oil of Juglans regia $\mathrm{L}$. and its constituents. Phytomedicine 19(13):1185-1190.

Rauha JP, Remes S, Heinonen M, Hopia A, Kähkönen M, Kujala T, Pihlaja K, Vuorela P. 2000. Antimicrobial effects of Finnish plant extracts containing flavonoids and other phenolic compounds. International Journal of Food Microbiology 56(1):3-12.

Salehzadeh A, Asadpour L, Naeemi AS, Houshmand E. 2014. Antimicrobial activity of methanolic extracts of Sambucus ebulus and Urtica dioica against clinical isolates of methicillin resistant Staphylococcus aureus. African Journal of Traditional, Complementary and Alternative Medicines 11(5):38-40.

Samuelsen AB. 2000. The traditional uses, chemical constituents, and biological activities of Plantago major L., A review, Journal of Ethnopharmacology 71(1):1-21.

Santos A, Barros L, Calhelha RC, Dueñas M, Carvalho AM, Santos-Buelga C, Ferreira IC. 2013. Leaves and decoction of Juglans regia L.: Different performances regarding bioactive compounds and in vitro antioxidant and antitumor effects. Industrial Crops and Products 51:430-436.

Sarac N, Ugur A. 2007. Antimicrobial activities and usage in folkloric medicine of some Lamiaceae species growing in Mugla, Turkey. EurAsian Journal of BioSciences 4:28-37.

Saraç DU, Özkan ZC, Akbulut S. 2013. Ethnobotanic features of Rize/Turkey province. Biodicon 6(3):57-66.

Sayah K, Marmouzi I, Naceiri Mrabti H, Cherrah Y, Faouzi MEA. 2017. Antioxidant activity and inhibitory potential of Cistus salviifolius (L.) and Cistus monspeliensis (L.) Aerial parts extracts against key enzymes linked to Hyperglycemia. BioMed Research International.

Serdar G, Sökmen M, Demir E, Sökmen A, Bektaş E. 2015. Extraction of antioxidative principles of Achillea biserrata M. Bieb. and chromatographic analyses. International Journal of Secondary Metabolite 2(2):3-15.

Shrivastava A. 2013. Ethnomedicinal plants used for treatment of gynecological disorders by tribal of Dindori district of Madhya Pradesh. International Journal of Pharmacy \& Life Sciences 4(12).

Shukla R, Chakravarty M, Gautam MP. 2008. Indigenous medicine used for treatment of gynecological disorders by tribal of Chhattisgarh, India. Journal of Medicinal Plants Research 2(12), 356-360.

Siatka T, Kašparová M. 2010. Seasonal variation in total phenolic and flavonoid contents and DPPH scavenging activity of Bellis perennis L. flowers. Molecules 15(12):9450-9461.

Singh B, Mall TP. 2011. Folk medicine used in gynecological disorders by rural population of Northwestern Tarai Forest of UP, India. Bioherald: International Journal of Biodiversity \& Environment 1(1). 
Stanisavljević IT, Stojičević SS, Veličković DT, Lazić ML, Veljković VB. 2008. Screening the antioxidant and antimicrobial properties of the extracts from plantain (Plantago major L.) leaves. Separation Science and Technology 43(14):3652-3662.

Steenkamp V. 2003. Traditional herbal remedies used by South African women for gynecological complaints. Journal of Ethnopharmacology 86(1):97-108.

Street RA, Sidana J, Prinsloo G. 2013. Cichorium intybus. traditional uses, phytochemistry, pharmacology, and toxicology, Evidence-Based Complementary and Alternative Medicine.

Tardío J, Pardo-de-Santayana M. 2008. Cultural importance indices: a comparative analysis based on the useful wild plants of Southern Cantabria (Northern Spain). Economic Botany 62(1):24-39.

Tawaha K, Alali FQ, Gharaibeh M, Mohammad M, El-Elimat T. 2007. Antioxidant activity and total phenolic content of selected Jordanian plant species. Food Chemistry 104(4):1372-1378.

Taye B, Giday M, Animut A, Seid J. 2011. Antibacterial activities of selected medicinal plants in traditional treatment of human wounds in Ethiopia. Asian Pacific Journal of Tropical Biomedicine 1(5):370-375.

Tetens I. 2015. Scientific Opinion on the safety of refined Buglossoides oil as a novel food ingredient. European Food Safety Authority.

Tetik F, Civelek S, Cakilcioglu U. 2013. Traditional uses of some medicinal plants in Malatya (Turkey). Journal of Ethnopharmacology 146:331-346.

TKH. 1990. Artvin ili Arazi Varliği Ankara: Tarim ve Köy Hizmetleri Genel Müdürlüğü Press.

Tomás-Menor L, Morales-Soto A, Barrajón-Catalán E, Roldán-Segura C, Segura-Carretero A, Micol V. 2013. Correlation between the antibacterial activity and the composition of extracts derived from various Spanish Cistus species. Food and Chemical Toxicology 55:313-322.

Tomczyk M, Pleszczyńska M, Wiater A. 2010. Variation in total polyphenolics contents of aerial parts of Potentilla species and their anticariogenic activity. Molecules 15(7):4639-4651.

Tomczyk M, Wiater A, Pleszczyńska M. 2011. In vitro anticariogenic effects of aerial parts of Potentilla recta and its phytochemical profile. Phytotherapy Research 25(3):343-350.

Trotter RT, Logan MH. 1986. Informant census: A new approach for identifying potentially effective medicinal plants. In: Etkin LN. (Ed.), Plants in indigenous medicine and diet. Routledge, Bedford Hill, NY, pp. 91-112.

Vidyasagar GM, Prashantkumar P. 2007. Traditional herbal remedies for gynecological disorders in women of Bidar district, Karnataka, India. Fitoterapia 78(1):48-51.

Wadankar GD, Malode SN, Sarambekar SL. 2011. Indigenous medicine used for treatment of gynecological and other related problems in Washim District, Maharashtra. International Journal of Pharmaceutical and Technical Research 3(2):698-701.

Wagner CS, De Gezelle J, Robertson M, Robertson K, Wilson M, Komarnytsky S. 2017. Antibacterial activity of medicinal plants from The Physicians of Myddvai, a 14th century Welsh medical manuscript. Journal of Ethnopharmacology 203:171-181.

Yadav JP, Kumar S, Siwach P. 2006. Folk medicine used in gynecological and other related problems by rural population of Haryana. Indian Journal of Traditional Knowledge 5(3):323-326.

Yildirim AB, Karakas FP, Turker AU. 2013. In vitro antibacterial and antitumor activities of some medicinal plant extracts, growing in Turkey. Asian Pacific Journal of Tropical Medicine 6(8):616-624.

Zhou J, Qu F. 2009. Treating gynecological disorders with traditional Chinese medicine: a review. African Journal of Traditional, Complementary and Alternative Medicines 6(4).

Žugić A, Đorđević S, Arsić I, Marković G, Živković J, Jovanović S, Tadić V. 2014. Antioxidant activity and phenolic compounds in 10 selected herbs from Vrujci Spa, Serbia. Industrial Crops and Products 52:519-527. 